JOURNAL OF THE

AMERICAN MATHEMATICAL SOCIETY

Volume 19, Number 2, Pages 447-459

S 0894-0347(05)00506-0

Article electronically published on September 26, 2005

\title{
THE BORDER RANK OF THE MULTIPLICATION OF $2 \times 2$ MATRICES IS SEVEN
}

\author{
J. M. LANDSBERG
}

\section{INTRODUCTION}

One of the leading problems of algebraic complexity theory is matrix multiplication. The naïve multiplication of two $n \times n$ matrices uses $n^{3}$ multiplications. In 1969, Strassen 20] presented an explicit algorithm for multiplying $2 \times 2$ matrices using seven multiplications. In the opposite direction, Hopcroft and Kerr [12 and Winograd 22] proved independently that there is no algorithm for multiplying $2 \times 2$ matrices using only six multiplications.

The precise number of multiplications needed to execute matrix multiplication (or any given bilinear map) is called the rank of the bilinear map. A related problem is to determine the border rank of matrix multiplication (or any given bilinear map), first introduced in [6, 5]. Roughly speaking, some bilinear maps may be approximated with arbitrary precision by less complicated bilinear maps and the border rank of a bilinear map is the complexity of arbitrarily small "good" perturbations of the map. These perturbed maps can give rise to fast exact algorithms for matrix multiplication; see [7. The border rank made appearances in the literature in the 1980s and early 1990s (see, e.g., 6, 5, 19, 8, 15, 2, 10, 11, 4, 3, 17, 16, 18, 1, 9, 21, ), but to our knowledge there has not been much progress on the question since then.

More precisely, for any complex projective variety $X \subset \mathbb{C P}^{N}=\mathbb{P} V$ and point $p \in \mathbb{P} V$, define the $X$-rank of $p$ to be the smallest number $r$ such that $p$ is in the linear span of $r$ points of $X$. Define $\sigma_{r}(X)$, the $r$-th secant variety of $X$, to be the Zariski closure of the set of points of $X$-rank $r$, and define the $X$-border rank of $p$ to be the smallest $r$ such that $p \in \sigma_{r}(X)$. The terminology is motivated by the case $X=\operatorname{Seg}\left(\mathbb{P}^{a-1} \times \mathbb{P}^{b-1}\right) \subset \mathbb{P}\left(\mathbb{C}^{a} \otimes \mathbb{C}^{b}\right)$, the Segre variety of rank one matrices. Then the $X$-rank of a matrix is just its usual rank.

Let $A^{*}, B^{*}, C$ be vector spaces and let $f: A^{*} \times B^{*} \rightarrow C$ be a bilinear map, i.e., an element of $A \otimes B \otimes C$. Let $X=\operatorname{Seg}(\mathbb{P} A \times \mathbb{P} B \times \mathbb{P} C) \subset \mathbb{P}(A \otimes B \otimes C)$ denote the Segre variety of decomposable tensors in $A \otimes B \otimes C$. The border rank of a bilinear map is its $X$-border rank. While for the Segre product of two projective spaces, border rank coincides with rank, here they can be quite different.

In this paper we prove the theorem stated in the title. Let $M$ Mult $\in \mathbb{C}^{4} \otimes \mathbb{C}^{4} \otimes$ $\mathbb{C}^{4}$ denote the matrix multiplication operator for $2 \times 2$ matrices. Strassen 18 ] showed that MMult $\notin \sigma_{5}\left(\mathbb{P}^{3} \times \mathbb{P}^{3} \times \mathbb{P}^{3}\right)$. Our method of proof is to decompose

Received by the editors August 23, 2004.

2000 Mathematics Subject Classification. Primary 68Q17.

Key words and phrases. Border rank, complexity of matrix multiplication, secant varieties.

(C)2005 American Mathematical Society Reverts to public domain 28 years from publication 
$\sigma_{6}\left(\mathbb{P}^{3} \times \mathbb{P}^{3} \times \mathbb{P}^{3}\right) \backslash \sigma_{5}\left(\mathbb{P}^{3} \times \mathbb{P}^{3} \times \mathbb{P}^{3}\right)$ into sixteen components, and then using caseby-case arguments, to show that MMult is not in any of the components. The decomposition rests upon a differential-geometric understanding of curves in submanifolds, which is carried out in $\S 2$. In $\S 3$ we roughly describe the components of $\sigma_{r}(X)$ for an arbitrary variety and give a precise description for $\sigma_{6}\left(\mathbb{P}^{3} \times \mathbb{P}^{3} \times \mathbb{P}^{3}\right)$, giving a normal form for a point of each component. Finally, in $\S 4$ we carry out our case-by-case analysis.

\section{TAYLOR SERIES FOR CURVES ON SUBMANIFOLDS}

Let $V$ be a vector space and $\mathbb{P} V$ the associated projective space of lines through the origin in $V$. If $x \in V$, we let $\hat{x} \subset V$ denote the line through $x$ and $[x] \in \mathbb{P} V$ the corresponding point in projective space. If $Z \subset \mathbb{P} V$ is a set, we let $\hat{Z} \subset V$ denote the corresponding cone in $V$.

We begin with some very general local differential geometry:

Lemma 2.1. Let $X \subset \mathbb{P} V$ be an analytic submanifold. Let $\left[x_{0}\right] \in X$ and choose a splitting $V=\hat{x}_{0} \oplus T_{\left[x_{0}\right]} X \oplus N_{\left[x_{0}\right]} X$. (This is necessary because in affine and projective geometry the tangent and normal spaces are only well defined as quotient spaces of $V$.) Having done so, we may and will identify abstract and embedded tangent spaces.

Let $x(t) \subset V$ be an analytic curve on $\hat{X}$ such that $x(0)=x_{0}$. Let $F_{j} \in$ $S^{j} T_{\left[x_{0}\right]}^{*} X \otimes N_{\left[x_{0}\right]} X$ denote the $j$-th Fubini form of $X$ at $\left[x_{0}\right]$ (see [13] $p .107$ for a definition or one can use the coordinate definition given in the proof below). Write, in local coordinates,

$$
x(t)=x_{0}+t x_{1}+t^{2} x_{2}+\cdots .
$$

Then there exists a sequence of elements $y_{1}, y_{2}, \ldots \in T_{\left[x_{0}\right]} X$ such that the following hold.

(i) $x_{1}=y_{1}$.

(ii) $x_{2}=F_{2}\left(y_{1}, y_{1}\right)+y_{2}$.

(iii) $x_{3}=F_{3}\left(y_{1}, y_{1}, y_{1}\right)+F_{2}\left(y_{1}, y_{2}\right)+y_{3}$.

(iv) In general,

$$
x_{k}=\sum_{j=2}^{k} \sum_{\substack{\lambda_{1}+2 \lambda_{2}+\cdots+f \lambda_{f}=k \\ \lambda_{1}+\cdots+\lambda_{f}=j}} F_{j}\left(\left(y_{1}\right)^{\lambda_{1}},\left(y_{2}\right)^{\lambda_{2}}, \ldots,\left(y_{f}\right)^{\lambda_{f}}\right)+y_{k} .
$$

Proof. First note that despite the choices of splittings, each term is well defined because of the lower order terms that appear with it. Also note that (ii) is well known in the classical geometry of surfaces, where $F_{2}=I I$ is the projective second fundamental form of $X$ at $\left[x_{0}\right]$. Take adapted coordinates $\left(w^{\alpha}, z^{\mu}\right)$ such that $\left[x_{0}\right]=$ $(0,0)$ and $T_{\left[x_{0}\right]} X$ is spanned by the first $n$ coordinates $(1 \leq \alpha \leq n)$. Then locally $X$ is given by equations

$$
z^{\mu}=f^{\mu}\left(w^{\alpha}\right)
$$

and

$$
F_{k}\left(\frac{\partial}{\partial w^{i_{1}}}, \ldots, \frac{\partial}{\partial w^{i_{k}}}\right)=\sum_{\mu} \frac{\partial^{k} f^{\mu}}{\partial w^{i_{1}}, \ldots, \partial w^{i_{k}}} \frac{\partial}{\partial z^{\mu}}
$$

Now write out the vectors $x_{j}$ in components, substitute into (1), and compare powers of $t$. The result follows. 
Let $\tau_{k+1}(X)$ denote the set of all points of the form $\left[x_{0}+x_{1}+\cdots+x_{k}\right]$, where the $x_{j}$ are as above, so $\tau_{1}(X)=X$ and $\tau_{2}(X)$ is the tangential variety of $X$, the union of all points on all tangent lines to $X$. Note that $\tau_{k}(X)$ is not the $k$ th osculating variety for $k>2$ and that the expected dimension of $\tau_{k}(X)$ is $k n$. Let $T_{k+1,\left[x_{0}\right]} X$ denote the set of all points of the form $\left[x_{0}+x_{1}+\cdots+x_{k}\right]$ so $\tau_{k+1}(X)=\bigcup_{\left[x_{0}\right] \in X} T_{k+1,\left[x_{0}\right]} X$.

\section{Components of $\sigma_{k}(X)$}

Let $X \subset \mathbb{P} V$ be a smooth projective variety. Let $\sigma_{k}^{0}(X)$ denote the set of points in $\sigma_{k}(X) \backslash \sigma_{k-1}(X)$ that may be written as the sum of $k$ points on $X$. Given $[p] \in \sigma_{k}(X)$, there exist analytic curves $p_{1}(t), \ldots, p_{k}(t)$ in $\hat{X}$, such that for $t \neq 0$, $[p(t)]:=\left[p_{1}(t)+\cdots+p_{k}(t)\right]$ is in $\sigma_{k}^{0}(X)$ and $[p(0)]=[p]$. This is true because in the Zariski topology a set that is open and dense is also dense in the classical topology. If one considers the set of honest secant $\mathbb{P}^{k-1}$ 's as a subset of the Grassmanian of all $\mathbb{P}^{k-1}$ 's in $\mathbb{P} V$, this set is Zariski open and dense in its Zariski closure and therefore open and dense as a subset of the Zariski closure in the analytic topology.

The point $p(0)$ is in the limiting $k$-plane corresponding to the first nonvanishing term in the Taylor series for $\left[p_{1}(t) \wedge p_{2}(t) \wedge \cdots \wedge p_{k}(t)\right]$, considered as a a point in the Grassmannian $G(k, V) \subset \mathbb{P}\left(\Lambda^{k} V\right)$. When taking a limit, there will be points $q_{1}, \ldots, q_{s}$ such that each of the $p_{j}$ 's limits to one of the $q_{\alpha}$ 's, e.g., $p_{1}(t), \ldots, p_{a_{1}}(t)$ limits to $q_{1}, p_{a_{1}+1}(t), \ldots, p_{a_{2}}(t)$ limits to $q_{2}$, etc. We consider separately the limits $L_{1}$ of $p_{1}(t) \wedge \cdots \wedge p_{a_{1}}(t)$, etc.

Now assume that $p_{i}(0)$ is a general point of $X$ for each $i$. (In particular, this assumption is automatic if $X$ is homogeneous.) We say the limiting $k$-plane is standard if $L_{1} \wedge L_{2} \wedge \cdots \wedge L_{s} \neq 0$, i.e., if we may consider the limiting linear spaces associated to each of the $q_{j}$ 's separately. Otherwise we say the limiting $k$-plane is exceptional. An example of an exceptional limit is when there are two limiting points $q_{1}, q_{2}$ but $q_{2}$ is in the tangent space of $q_{1}$. Another example of an exceptional limit is if at least three points go towards $q_{1}, q_{2} \in T_{3, q_{1}} X$ and $T_{3, q_{1}} X$ is not the entire ambient space.

3.1. Standard components for any variety. We first consider standard limits, so we may restrict our study to how $a$ curves $p_{1}(t), \ldots, p_{a}(t)$ can limit to a single point $x$. Write the Taylor series of $p_{j}(t)$ as

$$
p_{j}(t)=x+x_{1}^{j} t+x_{2}^{j} t^{2}+\cdots .
$$

Consider first for simplicity the case $a=3$. The first possible nonzero term is

$$
t^{2} x \wedge\left(x_{1}^{2} \wedge x_{1}^{3}-x_{1}^{1} \wedge x_{1}^{3}+x_{1}^{1} \wedge x_{1}^{2}\right) .
$$

If this term is nonzero, then $p \in \sigma_{k-1}(X)$ because any point on such a plane is also on a line of the form $x \wedge v$ with $v \in T_{[x]} X$ and so our three curves only really contribute as two. An easy exercise shows that if this term is zero, then either all three terms must be a multiple of one of them, say $x_{1}^{1}$, or two must be equal, say $x_{1}^{2}=x_{1}^{3}$. But the second case also leads to $p \in \sigma_{k-1}(X)$ when we examine the $t^{3}$ coefficient, so we must have all a multiple of $x_{1}^{1}$. So the only type of term we can have is the span of

$$
x, v, I I(v, v)+w
$$


where $v, w \in T_{[x]} X$. In other words, under the hypothesis that $p \notin \sigma_{k-1}(X)$, the only possible limit is a point of $\tau_{3}(X)$. Similarly for $a=4$, the only possible limit is a point of $\tau_{4}(X)$.

A new phenomenon occurs when $a=5$. We may obtain a point of $\tau_{5}(X)$ as above, but a second possibility occurs. Define $\tau_{5}(X)^{\prime}$ to be the union of all points in the span of

$$
x, x_{1}, I I\left(x_{1}, x_{1}\right)+x_{2}, y_{1}, I I\left(y_{1}, y_{1}\right)+y_{2},
$$

where $x_{1}, x_{2}, y_{1}, y_{2} \in T_{x} X$. In the notation above, this will occur if $x_{1}^{1}, x_{1}^{2}, x_{1}^{3}, x_{1}^{4}$ span a plane in $T_{[x]} X$.

For the case where all six points limit to the same point there are three possibilities. The first case yields $\tau_{6}(X)$. For the second, define $\tau_{6}(X)^{\prime}$ to be the union of all points in the span of $\left\langle x, x_{1}, I I\left(x_{1}, x_{1}\right)+x_{2}, F_{3}\left(x_{1}, x_{1}, x_{1}\right)+I I\left(x_{1}, x_{2}\right)+\right.$ $\left.x_{3}, y_{1}, I I\left(y_{1}, y_{1}\right)+y_{2}\right\rangle$ where $x_{1}, x_{2}, x_{3}, y_{1}, y_{2} \in T_{x} X$. For the third, define $\tau_{6}(X)^{\prime \prime}$ to be the union of all points in the span of $\left\langle x, x_{1}, I I\left(x_{1}, x_{1}\right)+x_{2}, F_{3}\left(x_{1}, x_{1}, x_{1}\right)+\right.$ $\left.I I\left(x_{1}, x_{2}\right)+x_{3}, I I\left(x_{1}, x_{1}\right)+y_{2}, F_{3}\left(x_{1}, x_{1}, x_{1}\right)+I I\left(x_{1}, y_{2}\right)+y_{3}\right\rangle$, where again the $x_{j}$ and $y_{j}$ are points of $T_{x} X$.

We now must take the span of $s$ such points. In general, given algebraic varieties $Y_{1}, \ldots, Y_{s} \subset \mathbb{P} V$, define their join $J\left(Y_{1}, \ldots, Y_{s}\right) \subset \mathbb{P} V$ to be the Zariski closure of the union of all $\mathbb{P}^{s-1}$ 's spanned by points $y_{1}, \ldots, y_{s}$ with $y_{j} \in Y_{j}$.

From the above discussion, we obtain all standard components of $\sigma_{6}(X) \backslash \sigma_{5}(X)$ where $X \subset \mathbb{P} V$ is any variety as follows (note that these components will not in general be disjoint):

(1) $\sigma_{6}^{0}(X)$

(2) $J\left(\sigma_{4}(x), \tau_{2}(X)\right)$,

(3) $J\left(\sigma_{3}(X), \tau_{3}(X)\right)$,

(4) $J\left(\tau_{3}(X), \tau_{3}(X)\right)$

(5) $J\left(\sigma_{2}(X), \tau_{2}(X), \tau_{2}(X)\right)$

(6) $J\left(\tau_{2}(X), \tau_{2}(X), \tau_{2}(X)\right)$,

(7) $J\left(\sigma_{2}(X), \tau_{4}(X)\right)$,

(8) $J\left(\tau_{2}(X), \tau_{4}(X)\right)$

(9) $J\left(X, \tau_{2}(X), \tau_{3}(X)\right)$

(10) $J\left(X, \tau_{5}(X)\right)$,

(11) $J\left(X, \tau_{5}(X)^{\prime}\right)$

(12) $\tau_{6}(X)$

(13) $\tau_{6}(X)^{\prime}$

(14) $\tau_{6}(X)^{\prime \prime}$

3.2. Standard components for $\operatorname{Seg}(\mathbb{P} A \times \mathbb{P} B \times \mathbb{P} C)$. In our case of

$$
X=\operatorname{Seg}(\mathbb{P} A \times \mathbb{P} B \times \mathbb{P} C),
$$

$X$ is not only homogenous, but it is a compact Hermitian symmetric space of rank three. Its only nonzero differential invariants are the second fundamental form $I I=$ $F_{2}$ and the third fundamental form $I I I$ (see [14, Theorem 4.1). The third fundamental form is the component of $F_{3}$ taking image in $N_{x} X / I I\left(S^{2} T_{[x]} X\right)$ (see [13, p. 96). Unlike the full $F_{3}$, it is a well-defined tensor $I I I \in S^{3} T_{x}^{*} X \otimes\left(N_{x} X / I I\left(S^{2} T_{[x]} X\right)\right)$. Similar to the situation in Lemma 2.1. we choose a splitting of $N_{x} X$ to make $I I I$ take values in a subspace of $N_{x} X$ instead of a quotient space.

Having such simple differential invariants makes it possible to have normal forms for elements of each standard component. We may write any element of $X$ as $p=$ 
$\left[a_{1} \otimes b_{1} \otimes c_{1}\right]=\left[a_{1} b_{1} c_{1}\right]$, where all vectors are nonzero. Here and in what follows, the $a_{j}$ 's are elements of $A, b_{j}$ 's of $B$, and $c_{j}$ 's of $C$, and we omit $\otimes$ in the notation for brevity. Any element of $T_{p} X$ may be written as $\left(a_{1} b_{1} c_{2}+a_{1} b_{2} c_{1}+a_{2} b_{1} c_{1}\right)$, where we allow the possibility of some (but not all) of $a_{2}, b_{2}, c_{2}$ to be zero.

If $p=\left[a_{1} b_{1} c_{1}\right]$ and $v=a_{1} b_{1} c_{2}+a_{1} b_{2} c_{1}+a_{2} b_{1} c_{1}, w=a_{1} b_{1} c_{3}+a_{1} b_{3} c_{1}+a_{3} b_{1} c_{1}, u=$ $a_{1} b_{1} c_{4}+a_{1} b_{4} c_{1}+a_{4} b_{1} c_{1} \in T_{p} X$, then, with the obvious choice of splitting,

$$
I I(v, w)=a_{1} b_{2} c_{3}+a_{1} b_{3} c_{2}+a_{2} b_{1} c_{3}+a_{3} b_{1} c_{2}+a_{2} b_{3} c_{1}+a_{3} b_{2} c_{1}
$$

and

$$
I I I(u, v, w)=a_{4} b_{2} c_{3}+a_{4} b_{3} c_{2}+a_{2} b_{4} c_{3}+a_{3} b_{4} c_{2}+a_{2} b_{3} c_{4}+a_{3} b_{2} c_{4} .
$$

Here are explicit normal forms for elements of each standard component when $X=\operatorname{Seg}(\mathbb{P} A \times \mathbb{P} B \times \mathbb{P} C)$. Repetitions and zeros among elements of the sets $\left\{a_{j}\right\}$, $\left\{b_{j}\right\}$, and $\left\{c_{j}\right\}$ are allowed as long as they do not force $p$ into $\sigma_{5}(X)$.

(1) $p=a_{1} b_{1} c_{1}+\cdots+a_{6} b_{6} c_{6} \in \sigma_{6}^{0}(X)$.

(2) $p=a_{1} b_{1} c_{1}+\cdots+a_{4} b_{4} c_{4}+a_{5} b_{5} c_{5}+\left(a_{5} b_{5} c_{6}+a_{5} b_{6} c_{5}+a_{6} b_{5} c_{5}\right) \in J\left(\sigma_{4}(x), \tau_{2}(X)\right)$.

(3) $p=a_{1} b_{1} c_{1}+a_{2} b_{2} c_{2}+a_{3} b_{3} c_{3}+a_{4} b_{4} c_{4}+\left(a_{4} b_{4} c_{5}+a_{4} b_{5} c_{4}+a_{5} b_{4} c_{4}\right)+\left[\left(a_{4} b_{4} c_{6}+\right.\right.$ $\left.\left.a_{4} b_{6} c_{4}+a_{6} b_{4} c_{4}\right)+2\left(a_{4} b_{5} c_{5}+a_{5} b_{4} c_{5}+a_{5} b_{5} c_{4}\right)\right] \in J\left(\sigma_{3}(X), \tau_{3}(X)\right)$.

(4) $p=a_{1} b_{1} c_{1}+\left(a_{1} b_{1} c_{2}+a_{1} b_{2} c_{1}+a_{2} b_{1} c_{1}\right)+\left[\left(a_{1} b_{1} c_{3}+a_{1} b_{3} c_{1}+a_{3} b_{1} c_{1}\right)+\right.$ $\left.2\left(a_{1} b_{2} c_{2}+a_{2} b_{1} c_{2}+a_{2} b_{2} c_{1}\right)\right]+a_{4} b_{4} c_{4}+\left(a_{4} b_{4} c_{5}+a_{4} b_{5} c_{4}+a_{5} b_{4} c_{4}\right)+\left[\left(a_{4} b_{4} c_{6}+\right.\right.$ $\left.\left.a_{4} b_{6} c_{4}+a_{6} b_{4} c_{4}\right)+2\left(a_{4} b_{5} c_{5}+a_{5} b_{4} c_{5}+a_{5} b_{5} c_{4}\right)\right] \in J\left(\tau_{3}(X), \tau_{3}(X)\right)$.

(5) $p=a_{1} b_{1} c_{1}+a_{2} b_{2} c_{2}+a_{3} b_{3} c_{3}+\left(a_{3} b_{3} c_{4}+a_{3} b_{4} c_{3}+a_{4} b_{3} c_{3}\right)+a_{5} b_{5} c_{5}+\left(a_{5} b_{5} c_{6}+\right.$ $\left.a_{5} b_{6} c_{5}+a_{6} b_{5} c_{5}\right) \in J\left(\sigma_{2}(X), \tau_{2}(X), \tau_{2}(X)\right)$.

(6) $p=a_{1} b_{1} c_{1}+\left(a_{1} b_{1} c_{2}+a_{1} b_{2} c_{1}+a_{2} b_{1} c_{1}\right)+a_{3} b_{3} c_{3}+\left(a_{3} b_{3} c_{4}+a_{3} b_{4} c_{3}+a_{4} b_{3} c_{3}\right)+$ $a_{5} b_{5} c_{5}+\left(a_{5} b_{5} c_{6}+a_{5} b_{6} c_{5}+a_{6} b_{5} c_{5}\right) \in J\left(\tau_{2}(X), \tau_{2}(X), \tau_{2}(X)\right)$.

(7) $p=a_{1} b_{1} c_{1}+a_{2} b_{2} c_{2}+a_{3} b_{3} c_{3}+\left(a_{3} b_{3} c_{4}+a_{3} b_{4} c_{3}+a_{4} b_{3} c_{3}\right)+\left[\left(a_{3} b_{3} c_{5}+a_{3} b_{5} c_{3}+\right.\right.$ $\left.\left.a_{5} b_{3} c_{3}\right)+2\left(a_{3} b_{4} c_{4}+a_{4} b_{3} c_{4}+a_{4} b_{4} c_{3}\right)\right]+\left[\left(a_{3} b_{3} c_{6}+a_{3} b_{6} c_{3}+a_{6} b_{3} c_{3}\right)+6 a_{4} b_{4} c_{4}+\right.$ $\left.\left(a_{3} b_{4} c_{5}+a_{3} b_{5} c_{4}+a_{4} b_{3} c_{5}+a_{5} b_{3} c_{4}+a_{4} b_{5} c_{3}+a_{5} b_{4} c_{3}\right)\right] \in J\left(\sigma_{2}(X), \tau_{4}(X)\right)$.

(8) $p=a_{1} b_{1} c_{1}+\left(a_{1} b_{1} c_{2}+a_{1} b_{2} c_{1}+a_{2} b_{1} c_{1}\right)+a_{3} b_{3} c_{3}+\left(a_{3} b_{3} c_{4}+a_{3} b_{4} c_{3}+a_{4} b_{3} c_{3}\right)+$ $\left[\left(a_{3} b_{3} c_{5}+a_{3} b_{5} c_{3}+a_{5} b_{3} c_{3}\right)+2\left(a_{3} b_{4} c_{4}+a_{4} b_{3} c_{4}+a_{4} b_{4} c_{3}\right)\right]+\left[\left(a_{3} b_{3} c_{6}+a_{3} b_{6} c_{3}+\right.\right.$ $\left.\left.a_{6} b_{3} c_{3}\right)+6 a_{4} b_{4} c_{4}+\left(a_{3} b_{4} c_{5}+a_{3} b_{5} c_{4}+a_{4} b_{3} c_{5}+a_{5} b_{3} c_{4}+a_{4} b_{5} c_{3}+a_{5} b_{4} c_{3}\right)\right] \in$ $J\left(\tau_{2}(X), \tau_{4}(X)\right)$.

(9) $p=a_{1} b_{1} c_{1}+a_{2} b_{2} c_{2}+\left(a_{2} b_{2} c_{3}+a_{2} b_{3} c_{2}+a_{3} b_{2} c_{2}\right)+a_{4} b_{4} c_{4}+\left(a_{4} b_{4} c_{5}+\right.$ $\left.a_{4} b_{5} c_{4}+a_{5} b_{4} c_{4}\right)+\left[\left(a_{4} b_{4} c_{6}+a_{4} b_{6} c_{4}+a_{6} b_{4} c_{4}\right)+2\left(a_{4} b_{5} c_{5}+a_{5} b_{4} c_{5}+a_{5} b_{5} c_{4}\right)\right] \in$ $J\left(X, \tau_{2}(X), \tau_{3}(X)\right)$.

(10) $p=a_{1} b_{1} c_{1}+a_{2} b_{2} c_{2}+\left(a_{2} b_{2} c_{3}+a_{2} b_{3} c_{2}+a_{3} b_{2} c_{2}\right)+\left[\left(a_{2} b_{2} c_{4}+a_{2} b_{4} c_{2}+a_{4} b_{2} c_{2}\right)+\right.$ $\left.2\left(a_{2} b_{3} c_{3}+a_{3} b_{2} c_{3}+a_{3} b_{3} c_{2}\right)\right]+\left[\left(a_{2} b_{2} c_{5}+a_{2} b_{5} c_{2}+a_{5} b_{2} c_{2}\right)+6 a_{3} b_{3} c_{3}+\left(a_{2} b_{3} c_{4}+\right.\right.$ $\left.\left.a_{2} b_{4} c_{3}+a_{3} b_{2} c_{4}+a_{4} b_{2} c_{3}+a_{3} b_{4} c_{2}+a_{4} b_{3} c_{2}\right)\right]+\left[\left(a_{2} b_{2} c_{6}+a_{2} b_{6} c_{2}+a_{6} b_{2} c_{2}\right)+\right.$ $2\left(a_{4} b_{3} c_{3}+a_{3} b_{4} c_{3}+a_{3} b_{3} c_{4}\right)+\left(a_{2} b_{3} c_{5}+a_{2} b_{5} c_{3}+a_{3} b_{2} c_{5}+a_{5} b_{2} c_{3}+a_{3} b_{5} c_{2}+\right.$ $\left.\left.a_{5} b_{3} c_{2}\right)+2\left(a_{2} b_{4} c_{4}+a_{4} b_{2} c_{4}+a_{4} b_{4} c_{2}\right)\right] \in J\left(X, \tau_{5}(X)\right)$.

(11) $p=a_{1} b_{1} c_{1}+a_{2} b_{2} c_{2}+\left(a_{2} b_{2} c_{3}+a_{2} b_{3} c_{2}+a_{3} b_{2} c_{2}\right)+\left[\left(a_{2} b_{2} c_{4}+a_{2} b_{4} c_{2}+a_{4} b_{2} c_{2}\right)+\right.$ $\left.2\left(a_{2} b_{3} c_{3}+a_{3} b_{2} c_{3}+a_{3} b_{3} c_{2}\right)\right]+\left(a_{2} b_{2} c_{5}+a_{2} b_{5} c_{2}+a_{5} b_{2} c_{2}\right)+\left[\left(a_{2} b_{2} c_{6}+a_{2} b_{6} c_{2}+\right.\right.$ $\left.\left.a_{6} b_{2} c_{2}\right)+2\left(a_{2} b_{5} c_{5}+a_{5} b_{2} c_{5}+a_{5} b_{5} c_{2}\right)\right] \in J\left(X, \tau_{5}(X)^{\prime}\right)$.

(12) $p=a_{1} b_{1} c_{1}+\left(a_{1} b_{1} c_{2}+a_{1} b_{2} c_{1}+a_{2} b_{1} c_{1}\right)+\left[\left(a_{1} b_{1} c_{3}+a_{1} b_{3} c_{1}+a_{3} b_{1} c_{1}\right)+\right.$ $\left.2\left(a_{1} b_{2} c_{2}+a_{2} b_{1} c_{2}+a_{2} b_{2} c_{1}\right)\right]+\left[\left(a_{1} b_{1} c_{4}+a_{1} b_{4} c_{1}+a_{4} b_{1} c_{1}\right)+6 a_{2} b_{2} c_{2}+\left(a_{1} b_{2} c_{3}+\right.\right.$ $\left.\left.a_{1} b_{3} c_{2}+a_{2} b_{1} c_{3}+a_{3} b_{1} c_{2}+a_{2} b_{3} c_{1}+a_{3} b_{2} c_{1}\right)\right]+\left[\left(a_{1} b_{1} c_{5}+a_{1} b_{5} c_{1}+a_{5} b_{1} c_{1}\right)+\right.$ $2\left(a_{2} b_{2} c_{3}+a_{2} b_{3} c_{2}+a_{3} b_{2} c_{2}\right)+\left(a_{1} b_{2} c_{4}+a_{1} b_{4} c_{2}+a_{2} b_{1} c_{4}+a_{4} b_{1} c_{2}+a_{2} b_{4} c_{1}+\right.$ $\left.\left.a_{4} b_{2} c_{1}\right)+2\left(a_{1} b_{3} c_{3}+a_{3} b_{1} c_{3}+a_{3} b_{3} c_{1}\right)\right]+\left[\left(a_{1} b_{1} c_{6}+a_{1} b_{6} c_{1}+a_{6} b_{1} c_{1}\right)+2\left(a_{2} b_{2} c_{4}+\right.\right.$ $\left.a_{2} b_{4} c_{2}+a_{4} b_{2} c_{2}\right)+2\left(a_{2} b_{3} c_{3}+a_{3} b_{2} c_{3}+a_{3} b_{3} c_{2}\right)+\left(a_{1} b_{2} c_{5}+a_{1} b_{5} c_{2}+a_{2} b_{1} c_{5}+\right.$ 
$\left.a_{5} b_{1} c_{2}+a_{2} b_{5} c_{1}+a_{5} b_{2} c_{1}\right)+\left(a_{1} b_{3} c_{4}+a_{1} b_{4} c_{3}+a_{3} b_{1} c_{4}+a_{4} b_{1} c_{3}+a_{3} b_{4} c_{1}+\right.$ $\left.\left.a_{4} b_{3} c_{1}\right)\right] \in \tau_{6}(X)$.

(13) $p=a_{1} b_{1} c_{1}+\left(a_{1} b_{1} c_{2}+a_{1} b_{2} c_{1}+a_{2} b_{1} c_{1}\right)+\left[\left(a_{1} b_{1} c_{3}+a_{1} b_{3} c_{1}+a_{3} b_{1} c_{1}\right)+\right.$ $\left.2\left(a_{1} b_{2} c_{2}+a_{2} b_{1} c_{2}+a_{2} b_{2} c_{1}\right)\right]+\left[\left(a_{1} b_{1} c_{4}+a_{1} b_{4} c_{1}+a_{4} b_{1} c_{1}\right)+6 a_{2} b_{2} c_{2}+\left(a_{1} b_{2} c_{3}+\right.\right.$ $\left.\left.a_{1} b_{3} c_{2}+a_{2} b_{1} c_{3}+a_{3} b_{1} c_{2}+a_{2} b_{3} c_{1}+a_{3} b_{2} c_{1}\right)\right]+\left(a_{1} b_{1} c_{5}+a_{1} b_{5} c_{1}+a_{5} b_{1} c_{1}\right)+$ $\left[\left(a_{1} b_{1} c_{6}+a_{1} b_{6} c_{1}+a_{6} b_{1} c_{1}\right)+2\left(a_{1} b_{5} c_{5}+a_{5} b_{1} c_{5}+a_{5} b_{5} c_{1}\right)\right] \in \tau_{6}(X)^{\prime}$.

(14) $p=a_{1} b_{1} c_{1}+\left(a_{1} b_{1} c_{2}+a_{1} b_{2} c_{1}+a_{2} b_{1} c_{1}\right)+\left[\left(a_{1} b_{1} c_{3}+a_{1} b_{3} c_{1}+a_{3} b_{1} c_{1}\right)+\right.$ $\left.2\left(a_{1} b_{2} c_{2}+a_{2} b_{1} c_{2}+a_{2} b_{2} c_{1}\right)\right]+\left[\left(a_{1} b_{1} c_{4}+a_{1} b_{4} c_{1}+a_{4} b_{1} c_{1}\right)+6 a_{2} b_{2} c_{2}+\left(a_{1} b_{2} c_{3}+\right.\right.$ $\left.\left.a_{1} b_{3} c_{2}+a_{2} b_{1} c_{3}+a_{3} b_{1} c_{2}+a_{2} b_{3} c_{1}+a_{3} b_{2} c_{1}\right)\right]+\left[\left(a_{1} b_{1} c_{5}+a_{1} b_{5} c_{1}+a_{5} b_{1} c_{1}\right)+\right.$ $\left.2\left(a_{1} b_{2} c_{2}+a_{2} b_{1} c_{2}+a_{2} b_{2} c_{1}\right)\right]+\left[\left(a_{1} b_{1} c_{6}+a_{1} b_{6} c_{1}+a_{6} b_{1} c_{1}\right)+6 a_{2} b_{2} c_{2}+\left(a_{1} b_{2} c_{5}+\right.\right.$ $\left.\left.a_{1} b_{5} c_{2}+a_{2} b_{1} c_{5}+a_{5} b_{1} c_{2}+a_{2} b_{5} c_{1}+a_{5} b_{2} c_{1}\right)\right] \in \tau_{6}(X)^{\prime \prime}$.

3.3. Exceptional components of $\sigma_{6}(\operatorname{Seg}(\mathbb{P} A \times \mathbb{P} B \times \mathbb{P} C))$. For an arbitrary variety, writing down all possible exceptional components is an intractible problem, but, again by the simplicity of the Segre variety, there are only two exceptional components of $\sigma_{6}(X) \backslash \sigma_{5}(X)$ and both occur in the case of two limit points.

To see this, say there are two exceptional limit points $p, q$ and all six curves limit to these. A priori the possible exceptional positions of $p$ and $q$ are that $p \in T_{q} X$ or that $p \in T_{k, q} X$, for some $k>1$. However, it is easy to see that an element of $T_{k, q} X$ cannot be decomposable unless either the "new" vector (denoted $y_{k}$ in the lemma) is zero, in which case we are reduced to a point of $\sigma_{5}(X)$, or all the Fubini forms occuring in the $k$-th term are zero and $y_{k}$ is decomposable-but in this case the $k$-th term is just a point of $T_{q} X$ and no new phenomenon occurs.

So say $p \in T_{q} X$. A prior $i$ there could be five different types of limits, depending on the number of curves that limit to $p$ and the number that limit to $q$. But $p \in T_{q} X$ implies $q \in T_{p} X$, so by symmetry we are reduced to three cases, five points limiting to $p$ and one to $q$, four to $p$ and two to $q$, and three to each of $p$ and $q$. Without loss of generality, take $p=a_{1} b_{1} c_{1}$ and $q=a_{1} b_{1} c_{2} \in T_{p} X$. If the $x_{1}$ term in the expansion for $p$ is not equal to $q$, then nothing new can occur as the expansions will not interfere with each other (in fact one ends up with a point of $\sigma_{5}(X)$ ).

In all cases, there is no ambiguity as to which terms in the Taylor expansions for $p$ and $q$ must contribute, as there is a unique choice of terms to wedge together that yield a term of lowest order.

Consider the case of five points limiting to $p$ and one to $q$. In order to get something new, we must have the first tangent vector to $p$ be $a_{1} b_{1} c_{2}$, as then we can use the first order term in the expansion of $q$. In this case we get a point of the following form, where 0's have been included where terms that ordinarily would not be zero are, e.g., the first zero represents $0=I I\left(a_{1} b_{1} c_{2}, a_{1} b_{1} c_{2}\right)$. Also for clarity a redundant $a_{1} b_{1} c_{2}$ is included in double parentheses.

$$
\begin{aligned}
& x=a_{1} b_{1} c_{1}+\left(a_{1} b_{1} c_{2}\right)+\left[\left(a_{1} b_{1} c_{3}+a_{1} b_{3} c_{1}+a_{3} b_{1} c_{1}\right)+0\right] \\
& +\left[\left(a_{1} b_{1} c_{4}+a_{1} b_{4} c_{1}+a_{4} b_{1} c_{1}\right)+0+\left(a_{1} b_{3} c_{2}+a_{3} b_{1} c_{2}\right)\right] \\
& +\left[\left(a_{1} b_{1} c_{5}+a_{1} b_{5} c_{1}+a_{5} b_{1} c_{1}\right)+0+\left(a_{1} b_{3} c_{3}+a_{3} b_{1} c_{3}+a_{3} b_{3} c_{1}\right)+\left(a_{1} b_{4} c_{2}+a_{4} b_{1} c_{2}\right)\right] \\
& +\left(\left(a_{1} b_{1} c_{2}\right)\right)+\left(a_{1} b_{1} c_{6}+a_{1} b_{6} c_{2}+a_{6} b_{1} c_{2}\right) .
\end{aligned}
$$

Here $x \in \tau_{5}(X) \subset \sigma_{5}(X)$, which can be seen by making the following substitutions: $\tilde{c}_{5}=c_{5}+c_{6}, \tilde{b}_{4}=b_{4}+b_{6}, \tilde{b}_{5}=b_{5}-b_{6}, \tilde{a}_{4}=a_{4}+a_{6}, \tilde{a}_{5}=a_{5}-a_{6}$. 
If the limit to $p$ is in $\tau_{5}(X)^{\prime}$, we obtain

$$
\begin{aligned}
x= & a_{1} b_{1} c_{1}+\left(a_{1} b_{1} c_{2}\right)+\left[\left(a_{1} b_{1} c_{3}+a_{1} b_{3} c_{1}+a_{3} b_{1} c_{1}\right)+0\right] \\
& +\left(a_{1} b_{1} c_{4}+a_{1} b_{4} c_{1}+a_{4} b_{1} c_{1}\right) \\
& +\left[\left(a_{1} b_{1} c_{5}+a_{1} b_{5} c_{1}+a_{5} b_{1} c_{1}\right)+\left(a_{1} b_{4} c_{4}+a_{4} b_{1} c_{4}+a_{4} b_{4} c_{1}\right)\right] \\
& +\left(\left(a_{1} b_{1} c_{2}\right)\right)+\left(a_{1} b_{1} c_{6}+a_{1} b_{6} c_{2}+a_{6} b_{1} c_{2}\right) .
\end{aligned}
$$

Here we may set $\tilde{c}_{5}=c_{5}+c_{3}, \tilde{b}_{5}=b_{5}+b_{3}, \tilde{a}_{5}=a_{5}+a_{3}$ to see that $x \in \sigma_{5}(X)$.

Still another possibility exists if in the cases above, the tangent vector to $q$, namely $\left(a_{1} b_{1} c_{6}+a_{1} b_{6} c_{2}+a_{6} b_{1} c_{2}\right)$, already appears as one of the terms in the expansion for $p$, in which case we get to examine another term in the Taylor series for $q$. This can happen when the limit to $p$ is a point of $\tau_{5}(X)$ and we have the coincidences $c_{6}=c_{4}, b_{6}=b_{3}, a_{6}=a_{3}, a_{4}, b_{4}=0$. Under these circumstances we get

$$
\begin{aligned}
x= & a_{1} b_{1} c_{1}+\left(a_{1} b_{1} c_{2}\right)+\left[\left(a_{1} b_{1} c_{3}+a_{1} b_{3} c_{1}+a_{3} b_{1} c_{1}\right)+0\right] \\
& +\left[\left(a_{1} b_{1} c_{4}\right)+0+\left(a_{1} b_{3} c_{2}+a_{3} b_{1} c_{2}\right)\right] \\
& +\left[\left(a_{1} b_{1} c_{5}+a_{1} b_{5} c_{1}+a_{5} b_{1} c_{1}\right)+0+\left(a_{1} b_{3} c_{3}+a_{3} b_{1} c_{3}+a_{3} b_{3} c_{1}\right)+0\right] \\
& +\left(\left(a_{1} b_{1} c_{2}\right)\right)+\left(\left(a_{1} b_{1} c_{4}+a_{1} b_{3} c_{2}+a_{3} b_{1} c_{2}\right)\right) \\
& +\left[\left(a_{7} b_{1} c_{2}+a_{1} b_{7} c_{2}+a_{1} b_{1} c_{7}\right)+\left(a_{1} b_{3} c_{4}+a_{3} b_{1} c_{4}+a_{3} b_{3} c_{2}\right)\right] .
\end{aligned}
$$

Call this case $E X_{1}$. If the limit to $p$ is a point of $\tau_{5}(X)^{\prime}$, there is no comparable term to cancel the term in the expansion for $q$.

One could try to make the $\left[\left(a_{7} b_{1} c_{2}+a_{1} b_{7} c_{2}+a_{1} b_{1} c_{7}\right)+\left(a_{1} b_{3} c_{4}+a_{3} b_{1} c_{4}+a_{3} b_{3} c_{2}\right)\right]$ term also appear in the limit for $p$, but this again forces too much degeneracy.

We have now examined all possibilities for five curves coming together to one point.

Now say the split between $p$ and $q$ is four/two. Again, if $q$ is not the initial tangent vector to $p$, we can get nothing new, and if it is, we obtain

$$
\begin{aligned}
x= & a_{1} b_{1} c_{1}+\left(a_{1} b_{1} c_{2}\right)+\left[\left(a_{1} b_{1} c_{3}+a_{1} b_{3} c_{1}+a_{3} b_{1} c_{1}\right)+0\right] \\
& +\left[\left(a_{1} b_{1} c_{4}+a_{1} b_{4} c_{1}+a_{4} b_{1} c_{1}\right)+0+\left(a_{1} b_{3} c_{2}+a_{3} b_{1} c_{2}\right)\right] \\
& +\left(\left(a_{1} b_{1} c_{2}\right)\right)+\left(a_{1} b_{1} c_{5}+a_{1} b_{5} c_{2}+a_{5} b_{1} c_{2}\right) \\
& +\left[\left(a_{1} b_{1} c_{6}+a_{1} b_{6} c_{2}+a_{6} b_{1} c_{2}\right)+2\left(a_{1} b_{5} c_{5}+a_{5} b_{1} c_{5}+a_{5} b_{5} c_{2}\right)\right] .
\end{aligned}
$$

Call this case $E X_{2}$.

Now consider the case where the tangent vector to $q$ occurs in the expansion for $p$ :

$$
\begin{aligned}
x= & a_{1} b_{1} c_{1}+\left(a_{1} b_{1} c_{2}\right)+\left[\left(a_{1} b_{1} c_{3}+a_{1} b_{3} c_{1}+a_{3} b_{1} c_{1}\right)+0\right] \\
& +\left[\left(a_{1} b_{1} c_{4}\right)+0+\left(a_{1} b_{3} c_{2}+a_{3} b_{1} c_{2}\right)\right] \\
& +\left(\left(a_{1} b_{1} c_{2}\right)\right)+\left(\left(a_{1} b_{1} c_{3}+a_{1} b_{3} c_{2}+a_{3} b_{1} c_{2}\right)\right) \\
& +\left[\left(a_{1} b_{1} c_{5}+a_{1} b_{5} c_{2}+a_{5} b_{1} c_{2}\right)+2\left(a_{1} b_{3} c_{2}+a_{3} b_{1} c_{4}+a_{3} b_{3} c_{2}\right)\right] \\
& +\left[a_{1} b_{1} c_{6}+a_{1} b_{6}+a_{1} b_{6} c_{2}+a_{6} b_{1} c_{2}+a_{4} b_{3} c_{4}+a_{1} b_{5} c_{4}+a_{5} b_{1} c_{4}\right. \\
& \left.+a_{1} b_{3} c_{5}+a_{3} b_{5} c_{2}+a_{5} b_{3} c_{2}\right] .
\end{aligned}
$$

Set $\tilde{c}_{6}=c_{6}+c_{4}, \tilde{c}_{5}=c_{5}+c_{2}$, and we see that $x \in \sigma_{5}(X)$. Higher order dengerations are similarly eliminated. 
Finally consider a 3-3 split. Assuming $q$ is the first tangent vector to $p$ in the expansion, we get

$$
\begin{aligned}
x= & a_{1} b_{1} c_{1}+\left(\left(a_{1} b_{1} c_{2}\right)\right)+\left[\left(a_{1} b_{1} c_{3}+a_{1} b_{3} c_{1}+a_{3} b_{1} c_{1}\right)+0\right] \\
& +\left[\left(a_{1} b_{1} c_{4}+a_{1} b_{4} c_{1}+a_{4} b_{1} c_{1}\right)+\left(a_{1} b_{3} c_{2}+a_{3} b_{1} c_{2}\right)\right] \\
& +\left(a_{1} b_{1} c_{2}\right)+\left(a_{1} b_{1} c_{5}+a_{1} b_{5} c_{2}+a_{5} b_{1} c_{2}\right) \\
& +\left[\left(a_{1} b_{1} c_{6}+a_{1} b_{6} c_{2}+a_{6} b_{1} c_{2}\right)+2\left(a_{1} b_{5} c_{5}+a_{5} b_{1} c_{5}+a_{5} b_{5} c_{2}\right)\right]
\end{aligned}
$$

Let $\tilde{b}_{3}=b_{3}+b_{4}, \tilde{b}_{6}=b_{6}+\tilde{b}_{3}, \tilde{a}_{3}=a_{3}+a_{4}, \tilde{a}_{6}=a_{6}+\tilde{a}_{3}$, and $\tilde{c}_{6}=c_{6}+c_{4}$, and we see that $x \in \sigma_{5}(X)$.

Assuming further coincidences similarly yields nothing new.

\section{Case-By-CASe arguments}

We will use variants of the proof of Proposition (17.9) in [7, which is due to Baur. Since there is a confusing reordering of indices in the proof in [7, we reproduce a proof here.

Theorem 4.1. If $A$ is a simple $k$-algebra, then the rank of the multiplication operator $M_{A}$ is $\geq 2 \operatorname{dim} A-1$.

Proof. Let $n:=\operatorname{dim} A$ and express $M_{A}$ optimally as $M_{A}=\alpha^{1} \otimes \beta^{1} \otimes c_{1}+\cdots+$ $\alpha^{r} \otimes \beta^{r} \otimes c_{r}$. Assume, to obtain a contradiction, that $r<2 \operatorname{dim} A-1$. (We switch notation, working in $A^{*} \otimes B^{*} \otimes C$, using $\alpha^{i}$ to denote elements of $A^{*}$ and $\beta^{i}$ to denote elements of $B^{*}=A^{*}$.)

By reordering if necessary, we may assume $\alpha^{1}, \ldots, \alpha^{n}$ is a basis of $A^{*}$. Let $b \in\left\langle\beta^{n}, \beta^{n+1}, \ldots, \beta^{r}\right\rangle^{\perp}$ be a nonzero element and consider the left ideal $A b$. We have $A b \subseteq\left\langle c_{1}, \ldots, c_{n-1}\right\rangle$. Let $L \supseteq A b$ be a maximal left ideal containing $A b$ and let $n-m=\operatorname{dim} L$. Since $\operatorname{dim} A b \geq m$ (the minimal dimension of an ideal), at least $m$ of the $\beta^{1}(b), \ldots, \beta^{n-1}(b)$ are nonzero (using again the linear independence of $\left.\alpha^{1}, \ldots, \alpha^{n}\right)$. Reorder among $1, \ldots, n-1$ such that the first $m$ are nonzero. We have $\left\langle c_{1}, \ldots, c_{m}\right\rangle \subseteq A b$ (by the linear independence of $\alpha^{1}, \ldots, \alpha^{m}$ ).

Note that $\beta^{m}, \ldots, \beta^{r}$ span $A^{*}$ (otherwise let $z \in\left\langle\beta^{m}, \ldots, \beta^{r}\right\rangle^{\perp}$ and consider the left ideal $A z$ which is too small). In particular, restricted to $L$, a subset of them spans $L^{*}$. We already know $\left.\beta^{m}\right|_{L} \neq 0$, so we use that as a first basis vector. Let $\beta^{i_{1}}, \ldots, \beta^{i_{n-m-1}}$ be a subset of $\beta^{m+1}, \ldots, \beta^{r}$ such that together with $\beta^{m}$, when restricted to $L$ they form a basis of $L^{*}$. Now let $j_{1}, \ldots, j_{r-n}$ be a complementary set of indices such that $\left\{i_{1}, \ldots, i_{n-m-1}\right\} \cup\left\{j_{1}, \ldots, j_{r-n}\right\}=\{m+1, \ldots, r\}$ and take $a \in\left\langle\alpha^{j_{1}}, \ldots, \alpha^{j_{r-n}}\right\rangle^{\perp}$ a nonzero element. Consider the right ideal $a A \subset$ $\left\langle c_{1}, \ldots, c_{m-1}, c_{m}, c_{i_{1}}, \ldots, c_{i_{n-m-1}}\right\rangle$. For any $y \in A$, there exists $w \in L$ such that $\beta^{m}(y)=\beta^{m}(w)$ and $\beta^{i_{f}}(y)=\beta^{i_{f}}(w)$ for $1 \leq f \leq n-m-1$, so ay $-a w \in$ $\left\langle c_{1}, \ldots, c_{m-1}\right\rangle$. But since $a w$ and the right-hand side are both included in $L$, we conclude $a A \subseteq L$, which is a contradiction as a left ideal cannot contain a nontrivial right ideal.

We now specialize to matrix multiplication of $2 \times 2$ matrices which we denote by $M M$ ult. Let $X=\operatorname{Seg}\left(\mathbb{P}^{3} \times \mathbb{P}^{3} \times \mathbb{P}^{3}\right)$. The theorem above implies $M M$ ult $\notin \sigma_{6}^{0}(X)$. We now show it is not in any of the other possiblilities. Let $A$ denote the algebra of $2 \times 2$ matrices. Note that all ideals of $A$ must be of dimension two.

Proposition 4.2. Mmult $\notin J\left(\sigma_{4}(X), \tau_{2}(X)\right), J\left(\sigma_{2}(X), \tau_{2}(X), \tau_{2}(X)\right)$ or $J\left(\tau_{2}(X)\right.$, $\left.\tau_{2}(X), \tau_{2}(X)\right)$. 
Proof. Say otherwise, i.e., that we had an expression, respectively,

$$
\begin{aligned}
\text { MMult }= & \alpha^{1} \beta^{1} c_{1}+\cdots+\alpha^{5} \beta^{5} c_{5}+\left(\alpha^{5} \beta^{5} c_{6}+\alpha^{5} \beta^{6} c_{5}+\alpha^{6} \beta^{5} c_{5}\right), \\
\text { MMult }= & \alpha^{1} \beta^{1} c_{1}+\alpha^{2} \beta^{2} c_{2}+\alpha^{3} \beta^{3} c_{3}+\left(\alpha^{3} \beta^{3} c_{4}+\alpha^{3} \beta^{4} c_{3}+\alpha^{4} \beta^{3} c_{3}\right)+\alpha^{5} \beta^{5} c_{5} \\
& +\left(\alpha^{5} \beta^{5} c_{6}+\alpha^{5} \beta^{6} c_{5}+\alpha^{6} \beta^{5} c_{5}\right), \\
\text { MMult }= & \alpha^{1} \beta^{1} c_{1}+\left(\alpha^{1} \beta^{1} c_{2}+\alpha^{1} \beta^{2} c_{1}+\alpha^{2} \beta^{1} c_{1}\right)+\alpha^{3} \beta^{3} c_{3} \\
& +\left(\alpha^{3} \beta^{3} c_{4}+\alpha^{3} \beta^{3} c_{4}+\alpha^{4} \beta^{3} c_{3}\right)+\alpha^{5} \beta^{5} c_{5}+\left(\alpha^{5} \beta^{5} c_{6}+\alpha^{5} \beta^{6} c_{5}+\alpha^{6} \beta^{5} c_{5}\right),
\end{aligned}
$$

which we refer to as the first, second, and third cases.

We first claim that $\alpha^{1}, \alpha^{2}, \alpha^{3}, \alpha^{4}$ or $\beta^{1}, \beta^{2}, \beta^{3}, \beta^{4}$ must be linearly independent. Otherwise, say both sets were dependent. Let $a^{\prime} \in\left\langle\alpha^{1}, \alpha^{2}, \alpha^{3}, \alpha^{4}\right\rangle^{\perp}, b^{\prime} \in$ $\left\langle\beta^{1}, \beta^{2}, \beta^{3}, \beta^{4}\right\rangle^{\perp}$. We have $a^{\prime} A=A b^{\prime}=\left\langle c_{5}, c_{6}\right\rangle$, a contradiction. The same conclusion holds for the 4-ples of vectors with indices 1,2,5,6 and those with indices $3,4,5,6$. (Here and in all arguments that follow, when we talk about finding vectors like $a, a^{\prime}, b, b^{\prime}$, etc., we mean nonzero vectors.)

We also note various independences among the $c_{j}$ 's. In case $3, c_{1}, c_{3}, c_{5}$ must be independent as otherwise consider $a \in\left\langle\alpha^{1}, \alpha^{3}, \alpha^{5}\right\rangle^{\perp}, b \in\left\langle\beta^{1}, \beta^{3}, \beta^{5}\right\rangle^{\perp}$. We have $a A \subseteq\left\langle c_{1}, c_{3}, c_{5}\right\rangle$ and in fact equality by the linear dependence, but similarly for $A b$. Thus $a A=A b$, a contradiction. In cases 1 and $2, c_{3}, c_{5}, c_{6}$ must be independent as otherwise in case 1 we could consider $a \in\left\langle\alpha^{1}, \alpha^{2}, \alpha^{4}\right\rangle^{\perp}$ and in case $2, a \in\left\langle\alpha^{1}, \alpha^{2}, \alpha^{3}\right\rangle^{\perp}$. In both cases we get $a A=\left\langle c_{3}, c_{5}, c_{6}\right\rangle$, but taking a corresponding $b$ yields a contradiction as above.

Cases 1 and 2: Without loss of generality, we assume $\alpha^{3}, \alpha^{4}, \alpha^{5}, \alpha^{6}$ are independent and consider $b \in\left\langle\beta^{1}, \beta^{2}, \beta^{4}\right\rangle^{\perp}$ in case 1 , and $b \in\left\langle\beta^{1}, \beta^{2}, \beta^{3}\right\rangle^{\perp}$ in case 2 . In both cases $A b \subset\left\langle c_{3}, c_{5}, c_{6}\right\rangle$. In case 2 we have the following matrix mapping the coefficients of $\alpha^{3}, \alpha^{5}, \alpha^{6}$ to the coefficients of $c_{3}, c_{5}, c_{6}$ :

$$
\operatorname{Mult}(\cdot, b)=\left(\begin{array}{ccc}
\beta^{4}(b) & 0 & 0 \\
0 & \beta^{5}(b)+\beta^{6}(b) & \beta^{5}(b) \\
0 & \beta^{5}(b) & 0
\end{array}\right)
$$

and in case 1 the matrix is the same except $\beta^{3}(b)$ replaces $\beta^{4}(b)$. By the linear indepence of $c_{3}, c_{5}, c_{6}$ and $\alpha^{3}, \alpha^{5}, \alpha^{6}$, the matrix must have rank two.

There are two subcases to consider depending on whether or not $\beta^{3}(b)=0$ in case 1 (resp. $\beta^{4}(b)=0$ in case 2 ).

Subcase 1: If $\beta^{3}(b)=0$ (resp. $\beta^{4}(b)=0$ ), then $\beta^{5}(b) \neq 0$ and $A b=\left\langle c_{5}, c_{6}\right\rangle$. We claim that $\beta^{1}, \beta^{2}, \beta^{4}, \beta^{5}$ (resp. $\left.\beta^{1}, \beta^{2}, \beta^{3}, \beta^{5}\right)$ is a basis of $A^{*}$ as otherwise let $b^{\prime} \in\left\langle\beta^{1}, \beta^{2}, \beta^{4}, \beta^{5}\right\rangle^{\perp}\left(\right.$ resp. $\left.b^{\prime} \in\left\langle\beta^{1}, \beta^{2}, \beta^{3}, \beta^{5}\right\rangle^{\perp}\right)$. We would have $A b^{\prime}=\left\langle c_{3}, c_{5}\right\rangle$, which has a one-dimensional intersection with $A b$, thus a contradiction. Thus at least one of $\beta^{1}, \beta^{2}, \beta^{4}$ (resp. $\beta^{1}, \beta^{2}, \beta^{3}$ ) together with $\beta^{5}$ restricted to $(A b)^{*}$ forms a basis of $(A b)^{*}$.

Say $\beta^{1}, \beta^{5}$ gives the basis. Then take $a \in\left\langle\alpha^{2}, \alpha^{3}, \alpha^{4}\right\rangle^{\perp}$. We have $a A \subset$ $\left\langle c_{1}, c_{5}, c_{6}\right\rangle$. But now for any $y \in A$ there exists $w \in A b$ such that $\beta^{1}(y)=\beta^{1}(w)$. Consider $a y-a w \subset\left\langle c_{5}, c_{6}\right\rangle=A b$. Since $a w \in A b$, we conclude $a y \in A b$ and thus $a A \subseteq A b$, a contradiction. The argument is the same if $\beta^{2}, \beta^{5}$ gives the basis; just change indices. In case 1 , the argument is still the same if $\beta^{4}, \beta^{5}$ gives the basis; again just change indices.

Say $\beta^{3}, \beta^{5}$ gives the basis in case 2 . Then take $a \in\left\langle\alpha^{1}, \alpha^{2}, \alpha^{3}\right\rangle^{\perp}$ so $a A \subseteq$ $\left\langle c_{3}, c_{5}, c_{6}\right\rangle$. Now use $\beta^{3}$ to show $a A \subseteq\left\langle c_{5}, c_{6}\right\rangle=A b$ to again obtain a contradiction. 
Subcase 2: If $\beta^{3}(b) \neq 0$ (resp. $\beta^{4}(b) \neq 0$ ), then $\beta^{5}(b)=0$ and $\beta^{6}(b) \neq 0$ and $A b=\left\langle c_{3}, c_{5}\right\rangle$. We claim that $\beta^{1}, \beta^{2}, \beta^{3}, \beta^{4}$ is a basis of $A^{*}$ as otherwise let $b^{\prime} \in$ $\left\langle\beta^{1}, \beta^{2}, \beta^{3}, \beta^{4}\right\rangle^{\perp}$ to get a notrivial intersection $A b \cap A b^{\prime}$ and a contradiction. Thus at least one of $\beta^{1}, \beta^{2}, \beta^{4}$ (resp. $\beta^{1}, \beta^{2}, \beta^{3}$ ) together with $\beta^{3}$ (resp. $\beta^{4}$ ) restricted to $(A b)^{*}$ forms a basis of $(A b)^{*}$. Each case leads to a contradiction as in the first subcase, finishing the proof for cases 1 and 2 .

Case 3: We first claim that $\alpha^{1}, \alpha^{3}, \alpha^{5}$ are linearly independent. Otherwise consider $a \in\left\langle\alpha^{1}, \alpha^{3}, \alpha^{5}, \alpha^{6}\right\rangle^{\perp}$ and $a^{\prime} \in\left\langle\alpha^{1}, \alpha^{3}, \alpha^{4}, \alpha^{5}\right\rangle^{\perp}$. We would have $a A=\left\langle c_{1}, c_{3}\right\rangle$, $a^{\prime} A=\left\langle c_{1}, c_{5}\right\rangle$ with a nontrivial intersection and thus a contradiction.

Now consider $b \in\left\langle\beta^{1}, \beta^{3}, \beta^{5}\right\rangle^{\perp}$, so $A b \subset\left\langle c_{1}, c_{3}, c_{5}\right\rangle$. We have the matrix

$$
\operatorname{Mult}(\cdot, b)=\left(\begin{array}{ccc}
\beta^{2}(b) & 0 & 0 \\
0 & \beta^{4}(b) & 0 \\
0 & 0 & \beta^{6}(b)
\end{array}\right)
$$

which must have rank two by the linear independence of $c_{1}, c_{3}, c_{5}$ and $\alpha^{1}, \alpha^{3}, \alpha^{5}$. By symmetry we may assume $\beta^{6}(b)=0$ so $A b=\left\langle c_{1}, c_{3}\right\rangle$ and $\beta^{1}, \beta^{3}, \beta^{5}, \beta^{6}$ are linearly dependent. We claim that $\beta^{1}, \beta^{2}, \beta^{3}, \beta^{5}$ gives a basis of $A^{*}$ as otherwise we could find a $b^{\prime} \in\left\langle\beta^{1}, \beta^{2}, \beta^{3}, \beta^{5}, \beta^{6}\right\rangle^{\perp}$ with $A b^{\prime}=\left\langle c_{3}\right\rangle$, a contradiction. Thus at least one of $\beta^{1}, \beta^{3}, \beta^{5}$ restricted to $(A b)^{*}$ together with $\beta^{2}$ gives a basis of $(A b)^{*}$.

Say $\beta^{1}, \beta^{2}$ gives a basis. Then take $a \in\left\langle\alpha^{3}, \alpha^{5}, \alpha^{6}\right\rangle^{\perp}$ so $a A \subset\left\langle c_{1}, c_{2}, c_{3}\right\rangle$. But $c_{2}$ appears in MMult with coefficient $\alpha^{1} \beta^{1}$ so we may argue as above to see $a A=$ $\left\langle c_{1}, c_{3}\right\rangle=A b$ to obtain a contradiction. We can argue similarly for $\beta^{3}, \beta^{2}$ using $a \in\left\langle\alpha^{1}, \alpha^{5}, \alpha^{6}\right\rangle^{\perp}$ and for $\beta^{5}, \beta^{2}$ using $a \in\left\langle\alpha^{1}, \alpha^{3}, \alpha^{5}\right\rangle^{\perp}$. This concludes the proof in case 3 .

Proposition 4.3. $M M u l t \notin J\left(\sigma_{3}(X), \tau_{3}(X)\right), J\left(\tau_{3}(X), \tau_{3}(X)\right), J\left(X, \tau_{2}(X), \tau_{3}(X)\right)$.

Proof. Assume otherwise that

$$
\begin{aligned}
\text { MMult }= & \alpha^{1} \beta^{1} c_{1}+\alpha^{2} \beta^{2} c_{2}+\alpha^{3} \beta^{3} c_{3}+\alpha^{4} \beta^{4} c_{4}+\left(\alpha^{4} \beta^{4} c_{5}+\alpha^{4} \beta^{5} c_{4}+\alpha^{5} \beta^{4} c_{4}\right) \\
& +\left(\alpha^{4} \beta^{4} c_{6}+\alpha^{4} \beta^{6} c_{4}+\alpha^{6} \beta^{4} c_{4}\right)+2\left(\alpha^{4} \beta^{5} c_{5}+\alpha^{5} \beta^{4} c_{5}+\alpha^{5} \beta^{5} c_{4}\right)
\end{aligned}
$$

for the first case and similarly for the other two cases. Let $b \in\left\langle\beta^{1}, \beta^{2}, \beta^{3}\right\rangle^{\perp}$ so $A b \subseteq\left\langle c_{4}, c_{5}, c_{6}\right\rangle$. Note that $c_{4}, c_{5}, c_{6}$ must be linearly independent, as otherwise take $a \in\left\langle\alpha^{1}, \alpha^{2}, \alpha^{3}\right\rangle^{\perp}$ and $a A=A b$.

Now consider the linear map $\operatorname{MMult}(\cdot, b)$. Assume for the moment that $\alpha^{4}, \alpha^{5}$, $\alpha^{6}$ are linearly independent. With respect to bases $c_{4}, c_{5}, c_{6}$ and $\alpha^{4}, \alpha^{5}, \alpha^{6}$, the map $\operatorname{MMult}(\cdot, b)$ has matrix

$$
\left(\begin{array}{ccc}
\beta^{4}(b)+\beta^{5}(b)+\beta^{6}(b) & \beta^{4}(b)+\beta^{5}(b) & \beta^{4}(b) \\
\beta^{4}(b)+\beta^{5}(b) & \beta^{4}(b) & 0 \\
\beta^{4}(b) & 0 & 0
\end{array}\right) .
$$

It must have a two-dimensional image, but this can occur only if $\beta^{4}(b)=0$, so we conclude $A b=\left\langle c_{4}, c_{5}\right\rangle$. But now take $a \in\left\langle\alpha^{1}, \alpha^{2}, \alpha^{3}\right\rangle^{\perp}$ and the same argument, assuming $\beta^{4}, \beta^{5}, \beta^{6}$ are linearly independent, gives $a A=\left\langle c_{4}, c_{5}\right\rangle$, a contradiction.

Now say $\beta^{4}, \beta^{5}, \beta^{6}$ fail to be linearly independent and consider the family of ideals $A b^{\prime}$ one obtains as $b^{\prime}$ ranges over $\left\langle\beta^{4}, \beta^{5}, \beta^{6}\right\rangle^{\perp}$. These ideals must all be contained in $\left\langle c_{1}, c_{2}, c_{3}\right\rangle$, hence they must be constant. Otherwise we would have two left ideals with a nontrivial intersection. But that means either $c_{1}, c_{2}, c_{3}$ fail to be linearly independent, which gives a contradiction as usual, or $\alpha^{1}, \alpha^{2}, \alpha^{3}$ fail 
to be linearly independent. But now if $\alpha^{1}, \alpha^{2}, \alpha^{3}$ fail to be linearly independent, consider $a^{\prime} \in\left\langle\alpha^{1}, \alpha^{2}, \alpha^{3}, \alpha^{4}\right\rangle^{\perp}$. We have $a^{\prime} A=\left\langle c_{4}, c_{5}\right\rangle$ equaling $A b$ above and giving a contradiction unless $\alpha^{4}, \alpha^{5}, \alpha^{6}$ also fail to be linearly independent.

Finally, assuming both $\alpha^{4}, \alpha^{5}, \alpha^{6}$ and $\beta^{4}, \beta^{5}, \beta^{6}$ are dependent, consider in the first two cases $\tilde{a} \in\left\langle\alpha^{3}, \alpha^{4}, \alpha^{5}, \alpha^{6}\right\rangle^{\perp}$ and $\tilde{b} \in\left\langle\beta^{3}, \beta^{4}, \beta^{5}, \beta^{6}\right\rangle^{\perp}$ and in the third case $\tilde{a} \in\left\langle\alpha^{2}, \alpha^{4}, \alpha^{5}, \alpha^{6}\right\rangle^{\perp}$ and $\tilde{b} \in\left\langle\beta^{2}, \beta^{4}, \beta^{5}, \beta^{6}\right\rangle^{\perp}$. In all cases we have $\tilde{a} A=A \tilde{b}=$ $\left\langle c_{1}, c_{2}\right\rangle$ and thus a contradiction.

Proposition 4.4. MMult $\notin J\left(\sigma_{2}(X), \tau_{4}(X)\right), J\left(\tau_{2}(X), \tau_{4}(X)\right)$.

Proof. We use normal forms as in $\S 3$. Consider $b \in\left\langle\beta^{1}, \beta^{2}, \beta^{3}\right\rangle^{\perp}$, so $A b \subset\left\langle c_{3}, c_{4}, c_{5}\right\rangle$. Assuming $\alpha^{3}, \alpha^{4}, \alpha^{5}$ are linearly independent, we get the same type of matrix as above and conclude $\beta^{4}(b)=0$ and $A b=\left\langle c_{3}, c_{4}\right\rangle$. If we also assume $\beta^{3}, \beta^{4}, \beta^{5}$ are linearly independent, taking $a \in\left\langle\alpha^{1}, \alpha^{2}, \alpha^{3}\right\rangle^{\perp}$, we have $a A=A b$, a contradiction.

If both $\alpha^{3}, \alpha^{4}, \alpha^{5}$ and $\beta^{3}, \beta^{4}, \beta^{5}$ are linearly dependent, we may take $a^{\prime} \in$ $\left\langle\alpha^{3}, \alpha^{4}, \alpha^{5}, \alpha^{6}\right\rangle^{\perp}$ and $b^{\prime} \in\left\langle\beta^{3}, \beta^{4}, \beta^{5}, \beta^{6}\right\rangle^{\perp}$ to get $a^{\prime} A=A b^{\prime}=\left\langle c_{1}, c_{2}\right\rangle$.

So assume $\beta^{3}, \beta^{4}, \beta^{5}$ are linearly dependent and $\alpha^{3}, \alpha^{4}, \alpha^{5}$ are independent. Consider the family of elements $b^{\prime \prime} \in\left\langle\beta^{3}, \beta^{4}, \beta^{5}\right\rangle^{\perp}$ so $A b^{\prime \prime} \subset\left\langle c_{1}, c_{2}, c_{3}\right\rangle$. This gives a family of left ideals in $\left\langle c_{1}, c_{2}, c_{3}\right\rangle$ but as above there must be just one ideal, which must be $\left\langle c_{1}, c_{2}\right\rangle$ as this is what one obtains for the element in $\left\langle\beta^{3}, \beta^{4}, \beta^{5}, \beta^{6}\right\rangle^{\perp}$. But this implies $\beta^{6}\left(b^{\prime \prime}\right)=0$ for all $b^{\prime \prime} \in\left\langle\beta^{3}, \beta^{4}, \beta^{5}\right\rangle^{\perp}$, i.e., $\beta^{6} \subset\left\langle\beta^{3}, \beta^{4}, \beta^{5}\right\rangle$, so now we may take in the first case of the proposition $b_{0} \in\left\langle\beta^{2}, \beta^{3}, \beta^{4}, \beta^{5}, \beta^{6}\right\rangle^{\perp}$ and $b_{0} \in\left\langle\beta^{1}, \beta^{3}, \beta^{4}, \beta^{5}, \beta^{6}\right\rangle^{\perp}$ in the second to obtain a one-dimensional left ideal $A b_{0}$.

Proposition 4.5. MMult $\notin J\left(X, \tau_{5}(X)\right)$.

Proof. Let $b \in\left\langle\beta^{1}, \beta^{2}, \beta^{3}\right\rangle^{\perp}$. Thus $A b \subseteq\left\langle c_{2}, c_{3}, c_{4}\right\rangle$. As usual we must have $c_{2}, c_{3}, c_{4}$ linearly independent. Considering the linear map $\operatorname{MMult}(\cdot, b)$, the only $\alpha^{j}$ 's that arise are $\alpha^{2}, \alpha^{3}, \alpha^{4}$ and, assuming they are linearly independent, the $3 \times 3$ matrix will have zero determinant only if $\beta^{4}(b)=0$, but then $A b=\left\langle c_{1}, c_{2}\right\rangle$.

Now consider $a \in\left\langle\alpha^{1}, \alpha^{2}, \alpha^{3}\right\rangle^{\perp}$. We see that if $\beta^{2}, \beta^{3}, \beta^{4}$ are linearly independent, we have $a A=A b$, a contradiction.

If say $\beta^{2}, \beta^{3}, \beta^{4}$ fail to be linearly independent, consider $b^{\prime} \in\left\langle\beta^{2}, \beta^{3}, \beta^{4}, \beta^{5}\right\rangle^{\perp}$ so $A b^{\prime}=\left\langle c_{1}, c_{2}\right\rangle$. Now consider $b^{\prime \prime} \in\left\langle\beta^{2}, \beta^{3}, \beta^{4}, \beta^{6}\right\rangle^{\perp}$. We have $A b^{\prime \prime} \subset\left\langle c_{1}, c_{2}, c_{3}\right\rangle$, so it has a nonzero intersection with $A b^{\prime}$, hence a contradiction unless it equals $A b^{\prime}$. But examining the matrix, even without assuming independence of $\alpha^{1}, \alpha^{2}, \alpha^{3}$, the only way that $A b^{\prime \prime}=A b^{\prime}$ is if $\beta^{5} \subset\left\langle\beta^{2}, \beta^{3}, \beta^{4}, \beta^{6}\right\rangle$, and if this occurs, we simply take $\tilde{b} \subset\left\langle\beta^{2}, \beta^{3}, \beta^{4}, \beta^{5}, \beta^{6}\right\rangle^{\perp}$ to obtain a one-dimensional ideal $A \tilde{b}=\left\langle c_{1}\right\rangle$.

Proposition 4.6. $M$ Mult $\notin \tau_{6}(X)$

Proof. Let $b \in\left\langle\beta^{1}, \beta^{2}, \beta^{3}\right\rangle^{\perp}$ and consider the ideal $A b \subset\left\langle c_{1}, c_{2}, c_{3}\right\rangle$. Again, $c_{1}, c_{2}, c_{3}$ must be linearly independent. If $\alpha^{1}, \alpha^{2}, \alpha^{3}$ are also linearly independent, then the only way for $A b$ to be two dimensional is if $\beta^{4}(b)=0$. Thus $A b=\left\langle c_{1}, c_{2}\right\rangle$. The same reasoning applied to $a \in\left\langle\alpha^{1}, \alpha^{2}, \alpha^{3}\right\rangle^{\perp}$ implies $a A=\left\langle c_{1}, c_{2}\right\rangle$, a contradiction, so at least one of the sets $\alpha^{1}, \alpha^{2}, \alpha^{3}, \beta^{1}, \beta^{2}, \beta^{3}$ must be linearly dependent.

Say just one set, e.g., $\beta^{1}, \beta^{2}, \beta^{3}$, fails to be linearly independent. Then by the reasoning above $\beta^{4} \subset\left\langle\beta^{1}, \beta^{2}, \beta^{3}\right\rangle$ because $\beta^{4}(b)=0$ for all $b \in\left\langle\beta^{1}, \beta^{2}, \beta^{3}\right\rangle^{\perp}$. But then there exists $b^{\prime} \in\left\langle\beta^{1}, \beta^{2}, \beta^{3}, \beta^{4}, \beta^{5}\right\rangle^{\perp}$ yielding an ideal $A b^{\prime}=\left\langle c_{2}\right\rangle$, a contradiction. 
Finally say both sets are dependent. Consider $b^{\prime \prime} \in\left\langle\beta^{1}, \beta^{2}, \beta^{3}, \beta^{4}\right\rangle^{\perp}$ and $a^{\prime \prime} \in$ $\left\langle\alpha^{1}, \alpha^{2}, \alpha^{3}, \alpha^{4}\right\rangle^{\perp}$. We have $A b^{\prime \prime}=a^{\prime \prime} A=\left\langle c_{1}, c_{2}\right\rangle$, a contradiction.

Proposition 4.7. MMult $\notin J\left(X, \tau_{5}(X)^{\prime}\right), \tau_{6}(X)^{\prime}, \tau_{6}(X)^{\prime \prime}, E X_{1}, E X_{2}$.

Proof. Consider $b \in\left\langle\beta^{1}, \beta^{2}, \beta^{3}\right\rangle^{\perp}$ in the first four cases and $b \in\left\langle\beta^{1}, \beta^{3}, \beta^{5}\right\rangle^{\perp}$ for the last. In all cases $A b$ is a fixed two-dimensional ideal (e.g. $\left\langle c_{2}, c_{5}\right\rangle$ in the first) but taking $a \in\left\langle\alpha^{1}, \alpha^{2}, \alpha^{3}\right\rangle^{\perp}$ in the first four cases and $a \in\left\langle\alpha^{1}, \alpha^{3}, \alpha^{5}\right\rangle^{\perp}$ for the last yields the same two-dimensional ideal, hence a contradiction.

\section{ACKNOWLEDGEMENTS}

The author was supported by NSF grant DMS-0305829. The key step for this paper was discovered while the author was a guest of L. Manivel at the University of Grenoble. He would like to thank L. Manivel for useful discussions and for providing him a respite from the climate in Atlanta. He also thanks the anonymous referee who gave a very careful reading to an earlier version of this paper.

\section{REFERENCES}

1. D. Bini, Border rank of a $p \times q \times 2$ tensor and the optimal approximation of a pair of bilinear forms, Automata, languages and programming (Proc. Seventh Internat. Colloq., Noordwijkerhout, 1980), Lecture Notes in Comput. Sci., vol. 85, Springer, Berlin, 1980, pp. 98-108. MR 0588996 (82e:68037)

2. D. Bini and M. Capovani, Tensor rank and border rank of band Toeplitz matrices, SIAM J. Comput. 16 (1987), no. 2, 252-258. MR0882531 (88g:68048)

3. Dario Bini, Tensor and border rank of certain classes of matrices and the fast evaluation of determinant inverse matrix and eigenvalues, Calcolo 22 (1985), no. 1, 209-228. MR0817042 (87g:65053)

4. , Border rank of $m \times n \times(m n-q)$ tensors, Linear Algebra Appl. 79 (1986), 45-51. MR0847189 (87j:15055)

5. Dario Bini, Milvio Capovani, Francesco Romani, and Grazia Lotti, $O\left(n^{2.7799}\right)$ complexity for $n \times n$ approximate matrix multiplication, Inform. Process. Lett. 8 (1979), no. 5, 234-235. MR0534068 (80h:68024)

6. Dario Bini, Grazia Lotti, and Francesco Romani, Approximate solutions for the bilinear form computational problem, SIAM J. Comput. 9 (1980), no. 4, 692-697. MR0592760 (82a:68065)

7. Peter Bürgisser, Michael Clausen, and M. Amin Shokrollahi, Algebraic complexity theory, Grundlehren der Mathematischen Wissenschaften [Fundamental Principles of Mathematical Sciences], vol. 315, Springer-Verlag, Berlin, 1997, with the collaboration of Thomas Lickteig. MR.1440179 (99c:68002)

8. Don Coppersmith and Shmuel Winograd, Matrix multiplication via arithmetic progressions, J. Symbolic Comput. 9 (1990), no. 3, 251-280. MR1056627(91i:68058)

9. J. W. de Bakker and J. van Leeuwen (eds.), Automata, languages and programming, Lecture Notes in Computer Science, vol. 85, Berlin, Springer-Verlag, 1980. MR0588986 (81i:68004)

10. H. F. de Groote, Lectures on the complexity of bilinear problems, Lecture Notes in Computer Science, vol. 245, Springer-Verlag, Berlin, 1987. MR0880703 (88d:68020)

11. B. Griesser, A lower bound for the border rank of a bilinear map, Calcolo 23 (1986), no. 2, 105-114. MR0897622 (88g:15021)

12. J. E. Hopcroft and L. R. Kerr, On minimizing the number of multiplications necessary for matrix multiplication, SIAM J. Appl. Math. 20 (1971), 30-36. MR0274293 (43:58)

13. Thomas A. Ivey and J. M. Landsberg, Cartan for beginners: differential geometry via moving frames and exterior differential systems, Graduate Studies in Mathematics, vol. 61, American Mathematical Society, Providence, RI, 2003. MR2003610 (2004g:53002)

14. Joseph M. Landsberg and Laurent Manivel, Construction and classification of complex simple Lie algebras via projective geometry, Selecta Math. (N.S.) 8 (2002), no. 1, 137-159. MR1890196 (2002m:17006) 
15. Thomas Lehmkuhl and Thomas Lickteig, On the order of approximation in approximative triadic decompositions of tensors, Theoret. Comput. Sci. 66 (1989), no. 1, 1-14. MR.1018840 (91f:68099)

16. Thomas Lickteig, A note on border rank, Inform. Process. Lett. 18 (1984), no. 3, 173-178. MR 0760371 (86c:68040)

17. Typical tensorial rank, Linear Algebra Appl. 69 (1985), 95-120. MR0798367 (87f:15017)

18. V. Strassen, Rank and optimal computation of generic tensors, Linear Algebra Appl. 52/53 (1983), 645-685. MR85b:15039

19. Degeneration and complexity of bilinear maps: some asymptotic spectra, J. Reine Angew. Math. 413 (1991), 127-180. MR1089800 (92m:11038)

20. Volker Strassen, Gaussian elimination is not optimal, Numer. Math. 13 (1969), 354-356. MR 0248973 (40:2223)

21. Algebraic complexity theory, Handbook of theoretical computer science, Vol. A, Elsevier, Amsterdam, 1990, pp. 633-672. MR.1127177

22. S. Winograd, On multiplication of $2 \times 2$ matrices, Linear Algebra and Appl. 4 (1971), 381-388. MR0297115 (45:6173)

Department of Mathematics, Texas A\& M University, College Station, Texas 778433368

E-mail address: jml@math.tamu.edu 